\title{
COMPUTER ALGORITHM FOR SOLVING OF THE CHAZY EQUATION OF THE THIRD ORDER WITH SIX SINGULAR POINTS
}

\author{
ALEXANDER CHICHURIN
}

Received 13 February, 2017

\begin{abstract}
The algorithm for calculating the coefficients of Chazy differential equation of the third order with six constant poles with respect to the unknown function is given. For such values of the poles a corresponding differential equation can be integrated in a symbolic form. When solving this problem the computational-algebraic algorithm to the construction of five non-linear differential equations of the third order, which are reduced to a linear inhomogeneous equations of the second order with six singular points is built. The algorithm is demonstrated on an example; five differential equations are obtained and their general solutions are found in elliptic functions. The calculations are implemented using Mathematica system.
\end{abstract}

2010 Mathematics Subject Classification: 34A25; 34A09; 70H03; 34A05

Keywords: Chazy differential equation, moveable singular points, general solution, elliptic functions

\section{INTRODUCTION}

The problem of finding third-order differential equations which solutions define new transcendental functions other than the Painleve transcendents [16], urged Chazy [4] to study the differential equations of the form

$$
y^{\prime \prime \prime}=R\left(y^{\prime \prime}, y^{\prime}, y, x\right),
$$

where $R$ a rational function of $y^{\prime \prime}, y^{\prime}, y$ with the analytic coefficients of $x$. The main problem, which is considered in the Chazy's fundamental paper [4], is the construction and study of new non-linear differential equations of the third order of P-type and in the description of new classes of transcendental functions defined by these equations. The study of the differential equations of the form (1.1), according to the Painleve method [16], reduces to the study of so-called "simplified" equations (a maximum number of poles in these equations is equal to six). Among the most interesting "simplified" equations Chazy emphasized a differential equation of the 
form

$$
y^{\prime \prime \prime}=\frac{P Q^{\prime \prime}-P^{\prime \prime} Q}{P Q^{\prime}-P^{\prime} Q} y^{\prime} y^{\prime \prime}-\frac{P^{\prime} Q^{\prime \prime}-P^{\prime \prime} Q^{\prime}}{P Q^{\prime}-P^{\prime} Q} y^{\prime 3},
$$

where $P, Q$ are fourth degree polynomials of $y$ with constant coefficients. For equation (1.2) the corresponding complete equation, according to the Painleve method, has the form [4]

$$
\begin{array}{r}
y^{\prime \prime \prime}=\sum_{k=1}^{6}\left(\left(y^{\prime}-a_{k}^{\prime}\right)\left(y^{\prime \prime}-a_{k}^{\prime \prime}\right)+A_{k}\left(y^{\prime}-a_{k}^{\prime}\right)^{3}+\right. \\
\left.B_{k}\left(y^{\prime}-a_{k}^{\prime}\right)^{2}+\mathrm{C}_{k}\left(y^{\prime}-a_{k}^{\prime}\right)\right) \times\left(y-a_{k}\right)^{-1}+ \\
D y^{\prime \prime}+E y^{\prime}+\prod_{i=1}^{6}\left(y-a_{i}\right) \sum_{k=1}^{6} \frac{F_{k}}{y-a_{k}},
\end{array}
$$

32 coefficients $a_{k}, A_{k}, B_{k}, \mathrm{C}_{k}, F_{k}(k=\overline{1,6}), D, E$ satisfy the system, consisting of 31 algebraic and differential equations [4] (Application, systems (5.1)-(5.6)).

Chazy has not completed integration of the system (5.1)-(5.6), and has not obtained the equations of the form (1.3), that was primarily due to the necessity of carrying out cumbersome symbolic computations and transformations. He only showed that some cases of degeneration of this equation are the canonical Painleve equations [11].

Research in the system (5.1) was conducted in the works [13,14], and its solution has the form $[8,14]$

$$
\begin{aligned}
& A_{k}=\frac{3 \beta_{3}-6 a_{k}^{4}+4 \sigma_{1} a_{k}^{3}+3\left(\alpha_{2}-\sigma_{2}\right) a_{k}^{2}-3 \beta_{2} a_{k}-\sigma_{4}}{6 a_{k}^{5}-5 \sigma_{1} a_{k}^{4}+4 \sigma_{2} a_{k}^{3}-3 \sigma_{3} a_{k}^{2}+2 \sigma_{4} a_{k}-\sigma_{5}}, \\
& \beta_{2}=\frac{\left(3 \alpha_{2}-\sigma_{2}\right)\left(2 \alpha_{2} \sigma_{1}-3 \sigma_{3}\right)-2 \sigma_{1} \sigma_{4}+6 \sigma_{5}}{\sigma_{1}^{2}+18 \alpha_{2}-6 \sigma_{2}}, \\
& \beta_{3}=\frac{\left(\sigma_{2}-3 \alpha_{2}\right)\left(12 \alpha_{2}^{2}-4 \sigma_{2} \alpha_{2}+\sigma_{1} \sigma_{3}-4 \sigma_{4}\right)+2 \sigma_{1} \sigma_{5}}{2\left(\sigma_{1}^{2}+18 \alpha_{2}-6 \sigma_{2}\right)},
\end{aligned}
$$


where $\sigma_{k}$ are basic symmetric polynomials, consisting of elements $a_{k}(k=\overline{1,6})$, function $\alpha_{2}$ satisfies the equation of the fifth degree

$$
\begin{aligned}
& 1296 \alpha_{2}^{5}-1296 \sigma_{2} \alpha_{2}^{4}+\left(432 \sigma_{2}^{2}+216 \sigma_{1} \sigma_{3}-\right. \\
& \left.432 \sigma_{4}\right) \alpha_{2}^{3}-\left(48 \sigma_{2}^{3}+144 \sigma_{1} \sigma_{3} \sigma_{2}-288 \sigma_{4} \sigma_{2}-\right. \\
& \left.24 \sigma_{1}^{2} \sigma_{4}+216 \sigma_{1} \sigma_{5}-1296 \sigma_{6}\right) \alpha_{2}^{2}+ \\
& \left(9 \sigma_{3}^{2} \sigma_{1}^{2}-4 \sigma_{5} \sigma_{1}^{3}-8 \sigma_{2} \sigma_{4} \sigma_{1}^{2}+144 \sigma_{6} \sigma_{1}^{2}+\right. \\
& \left(24 \sigma_{2}^{2} \sigma_{3}-36 \sigma_{3} \sigma_{4}+72 \sigma_{2} \sigma_{5}\right) \sigma_{1}-48 \sigma_{2}^{2} \sigma_{4}+ \\
& \left.108 \sigma_{3} \sigma_{5}-864 \sigma_{2} \sigma_{6}\right) \alpha_{2}-3 \sigma_{1}^{2} \sigma_{2} \sigma_{3}^{2}-8 \sigma_{1}^{2} \sigma_{4}^{2}- \\
& 72 \sigma_{5}^{2}+2 \sigma_{1}^{3} \sigma_{3} \sigma_{4}+12 \sigma_{1} \sigma_{2} \sigma_{3} \sigma_{4}-6 \sigma_{1}^{2} \sigma_{3} \sigma_{5}- \\
& 36 \sigma_{2} \sigma_{3} \sigma_{5}+48 \sigma_{1} \sigma_{4} \sigma_{5}+4 \sigma_{1}^{4} \sigma_{6}+ \\
& 144 \sigma_{2}^{2} \sigma_{6}-48 \sigma_{1}^{2} \sigma_{2} \sigma_{6}=0
\end{aligned}
$$

and the denominator in the relations (1.4) $\sigma_{1}^{2}+18 \alpha_{2}-6 \sigma_{2}$ is not equal to zero. If the condition

$$
\sigma_{1}^{2}+18 \alpha_{2}-6 \sigma_{2}=0
$$

holds, then the values $A_{k}$ have the form

$$
\begin{gathered}
A_{k}=-\frac{6 a_{k}-\sigma_{1}}{108} \times \\
\frac{108 a_{k}^{3}-54 \sigma_{1} a_{k}^{2}-6\left(\sigma_{1}^{2}-6 \sigma_{2}\right) a_{k}-\sigma_{1}^{3}-108 \alpha_{3}+6 \sigma_{1} \sigma_{2}-27 \sigma_{3}}{6 a_{k}^{5}-5 \sigma_{1} a_{k}^{4}+4 \sigma_{2} a_{k}^{3}-3 \sigma_{3} a_{k}^{2}+2 \sigma_{4} a_{k}-\sigma_{5}}
\end{gathered}
$$

and the equality still preserves

$$
\begin{gathered}
\sigma_{6}=\frac{1}{2} \alpha_{3}\left(4 \alpha_{3}+\sigma_{3}\right)+\frac{\sigma_{1}^{2}-6 \sigma_{2}}{5832} \times \\
\left(\sigma_{1}\left(\sigma_{1}^{3}-6 \sigma_{2} \sigma_{1}+108 \alpha_{3}+27 \sigma_{3}\right)-108 \sigma_{4}\right) .
\end{gathered}
$$

If the coefficients $a_{k}$ are constants and $D=0$, then equation (1.3) is simplified

$$
y^{\prime \prime \prime}=\sum_{k=1}^{6} \frac{y^{\prime} y^{\prime \prime}+A_{k} y^{\prime 3}+\mathrm{C}_{k} y^{\prime}}{y-a_{k}}+E y^{\prime} .
$$

In the papers $[5,8,9]$ by using solutions (1.4), (1.7) the new solutions of the equation (1.9) were found and properties of such solutions were also studied. 
Let us note that the coefficients $a_{k}, A_{k}, \mathrm{C}_{k}, E$ of the equation (1.9) connected by the relations $[4,8,13]$

$$
\sum_{\substack{k=1 \\ j \neq k}}^{6} \frac{\mathrm{C}_{j}-\mathrm{C}_{k}}{a_{k}-a_{j}}=2 A_{k}+E \quad(k, j=\overline{1,6}) .
$$

The number of equations of the kind (1.9) is equal five, since the equation (1.5) has five, in the general case, complex roots. The essential fact is that for some sets of values of coefficients $a_{k}$ the roots of the equation (1.5) in radicals can be found (this case is implemented in the example).

In those cases where the roots of the equation (1.5) can't be found in the radicals, the coefficients $A_{k}, \mathrm{C}_{k}$ are calculated only approximately. It's worthy to note that computer algebra systems have built-in functions that allow saving the symbolic form of the coefficients $A_{k}, \mathrm{C}_{k}$ and calculate them with a given accuracy (for example, the function Root-object [1]). For the differential equations built with such approximated coefficients the integration procedure used in this paper can be applied as well.

Note that the differential equation (1.3) can be studied by constructing an equivalent system of two differential equations. In paper [4] Chazy built such a system. Other equivalent systems were built by N.A. Lukashevich [12]. For equation (1.9) the equivalent systems were built in the papers $[10,18]$. Here the coefficients of the obtained systems have been found with the help of an analytical method, which was implemented as a software module.

Study of the equation (1.3) can be carried out also by using the approximate method proposed by V.N. Orlov [15]. This method is based on the separation of the domain of construction solutions into a regular area and into neighborhoods of movable critical points, and on the construction of a sequence of analytic continuations.

The method of constructing one- and two-parameter families of solutions of the equation (1.3) for certain values of coefficients is considered in [6,7].

An important circumstance facilitating the study of the equation (1.9) is that by substituting

$$
\frac{d y}{d x}=\sqrt{z(y)},
$$

proposed by N.A.Lukashevich [13], this equation can be linearized. Indeed, as shown in [8] equation (1.9) reduced to the linear equation of the second order with six different poles with respect to the independent variable

$$
\frac{d^{2} z}{d y^{2}}=\sum_{i=1}^{6} \frac{1}{y-a_{i}} \frac{d z}{d y}+2 \sum_{i=1}^{6} \frac{A_{i}}{y-a_{i}} z+2 \sum_{i=1}^{6} \frac{\mathrm{C}_{i}}{y-a_{i}}+E .
$$

In the paper [8] proved that the solution of equation (1.9) with coefficients that satisfy the relations (1.4) - (1.8) and the system (1.10), is constructed by means of the 
quadrature with using the inverse value of the general solution of equation (1.12). For the certain values of the coefficients $a_{k}$ the general solution is written by means of elliptic functions, and in the simplest cases - even by means of elementary functions.

In this paper we propose a computational algorithm for constructing the Chazy equation of the form (1.9) with coefficients satisfying the relations (1.4) - (1.8), (1.10). This equation can be integrated in the symbolic form and depends on the set values of the six coefficients $a_{k}([8,9,17])$. The integration of the obtained equations is demonstrated by the example. All analytical transformations and numerical calculations are performed using computer algebra system Mathematica.

\section{AN ALGORITHM FOR CONSTRUCTING EQUATIONS AND THEIR INTEGRATION}

The proposed algorithm consists of several steps.

a) We determine values of the coefficients $a_{k}$ and calculate the values of the basic symmetric polynomials $\sigma_{k}$, composed of them. After this we find five roots $\alpha_{2 i}$ $(i=\overline{1,5})$ of the equation (1.5).

b) Using formulas (1.4) or (1.7)-(1.8) with the condition (1.6) for the given values $a_{k}$ and $\alpha_{2 i}$ we find the corresponding values of the coefficients $A_{k}(k=\overline{1,6})$.

c) For each from the five found sets of values $a_{k}, A_{k}(k=\overline{1,6})$, we solve system (1.10) with respect to unknowns $\mathrm{C}_{k}$; system (1.10) is degenerate and therefore we will solve it for the unknown $\mathrm{C}_{j}(j=\overline{3,6})$, which will depend on the values of $\mathrm{C}_{1}$, $\mathrm{C}_{2}, E$ ).

d) We substitute the obtained values $a_{k}, A_{k}(k=\overline{1,6})$ and values $\mathrm{C}_{j}(j=\overline{3,6})$ for each of the roots $\alpha_{2 i}(i=\overline{1,5})$ into equation (1.9). As a result, we obtain five differential equations.

e) Using substitution (1.11) we linearize each of the five equations and write them as equation (1.12). If the coefficient of $E$ is constant, then the resulting linear equation can be integrated. Then, using the inverse of (1.11) substitution, we find a general solution for each of the five equations in the form (1.9). As a result, we obtain families of functions defined in the implicit form; they contain three parameters $C_{1}, C_{2}$, $E$ and three arbitrary constants $c_{i}(i=1,2,3)$. If the coefficient of $E$ is not constant, it is required in each case to check the integrability of the resulting linear equation. If the linear equation can be integrated, then we find a general solution of equation (1.9) and use the inverse substitution for (1.11).

\section{EXAMPLE}

Algorithm for constructing equations presented above and their integration we demonstrate by the example. We choose the values of $a_{k}(k=\overline{1,6})$ such that the roots of the equation (1.5) can be found by using radicals.

Consider the equation (1.9) where $E=$ const with coefficients of the form

$$
a_{1}=1, a_{2}=-1, a_{3}=2, a_{4}=-2, a_{5}=\frac{1}{2}, a_{6}=-\frac{1}{2} .
$$


Follow the step a) of algorithm, we obtain a polynomial

$$
1296 \alpha_{2}^{5}+27216 \alpha_{2}^{4}+154224 \alpha_{2}^{3}-146448 \alpha_{2}^{2}-2939328 \alpha_{2}-4064256 .
$$

The roots of the polynomial (3.2) $\alpha_{2}$ have the form

$$
1,-\frac{\sqrt{57}+11}{8}, \frac{\sqrt{57}-11}{8},-\frac{7}{4},
$$

and the root $-\frac{7}{4}$ is a double and satisfies the equation (1.6). Note that the first three roots do not satisfy the equation (1.6), therefore, to calculate coefficients $A_{k}$ ( $k=$ $\overline{1,6}$ ) we use formulas (1.4).

For root $\alpha_{21}=1$ we find the following values of the coefficients:

$$
\begin{aligned}
& A_{1}=-1, A_{2}=1, A_{3}=-\frac{13}{20}, \\
& A_{4}=\frac{13}{20}, A_{5}=-\frac{7}{5}, A_{6}=\frac{7}{5}, \\
& \mathrm{C}_{3}=\frac{45 \mathrm{C}_{1}+19 \mathrm{C}_{2}+24 E}{40}, \mathrm{C}_{4}=\frac{19 \mathrm{C}_{1}+45 \mathrm{C}_{2}-24 E}{40}, \\
& \mathrm{C}_{5}=\frac{18 \mathrm{C}_{1}-10 \mathrm{C}_{2}-3 E}{20}, \mathrm{C}_{6}=\frac{18 \mathrm{C}_{2}-10 \mathrm{C}_{1}+3 E}{20},
\end{aligned}
$$

where $\mathrm{C}_{1}, \mathrm{C}_{2}, E$ are arbitrary constants. Equation (1.9) with coefficients (3.1), (3.4) reduced by the substitution (1.11) to the equation (1.12) of the form

$$
\begin{aligned}
& 3 \mathrm{C}_{1}(y+1)^{2}\left(8 y^{3}-8 y^{2}-14 y+11\right)+ \\
& 3 \mathrm{C}_{2}(y-1)^{2}\left(8 y^{3}+8 y^{2}-14 y-11\right)+ \\
& \left(8 y^{6}-24 y^{4}+24 y^{2}-8\right) E- \\
& \left(48 y^{4}-150 y^{2}+66\right) z+ \\
& \left(24 y^{5}-84 y^{3}+42 y\right) z^{\prime}- \\
& \left(4 y^{6}-21 y^{4}+21 y^{2}-4\right) z^{\prime \prime}=0 .
\end{aligned}
$$

Equation (3.5) is linear nonhomogeneous differential equation of the second order with variable coefficients. Integration of this equation can be performed by the classical method. For this we integrate the first corresponding homogeneous equation, then by using quadratures we find its solution. These calculations are implemented by using computer algebra systems (for example, using the function DSolve [2, 17] in Mathematica system). One can also use the search algorithm of rational solutions of linear ordinary differential equations with polynomial coefficients presented in the work [3]. The general solution of equation (3.5) has the form

$$
\begin{aligned}
& z=\frac{1}{2}\left(\mathrm{C}_{1}(2 y+1)+\mathrm{C}_{2}(2 y-1)+\right. \\
& \left.\left.2\left(c_{1}\left(2 y^{2}-5 y+2\right)\right)^{2}+y\left(c_{2} y^{2}+c_{2}+E y\right)\right)\right),
\end{aligned}
$$

where $c_{1}, c_{2}$ are arbitrary constants. Substituting the expression (3.6) into formula (1.11), and integrating the resulting equation, we find the general solution of equation 
(1.9), (3.1), (3.4) implicitly

$$
\begin{aligned}
& \frac{2 F\left(\arcsin \left(\sqrt{\frac{\left(\psi_{2}-\psi_{4}\right)\left(\psi_{1}-y(x)\right)}{\left(\psi_{1}-\psi_{4}\right)\left(\psi_{2}-y(x)\right)}}\right) \mid \frac{\left(\psi_{2}-\psi_{3}\right)\left(\psi_{1}-\psi_{4}\right)}{\left(\psi_{1}-\psi_{3}\right)\left(\psi_{2}-\psi_{4}\right)}\right)}{\left(\psi_{1}\right) \sqrt{z}} \times \\
& \frac{\left(\psi_{1}-y(x)\right) \sqrt{\frac{\left(\psi_{1}-\psi_{2}\right)\left(\psi_{3}-y(x)\right)}{\left(\psi_{1}-\psi_{3}\right)\left(\psi_{2}-y(x)\right)}}\left(\psi_{4}-y(x)\right)}{\sqrt{\frac{\left(\psi_{1}-\psi_{2}\right)\left(\psi_{2}-\psi_{4}\right)\left(\psi_{1}-y(x)\right)\left(\psi_{4}-y(x)\right)}{\left(\psi_{1}-\psi_{4}\right)^{2}\left(\psi_{2}-y(x)\right)^{2}}}}=\frac{x}{\sqrt{2}}+c_{3},
\end{aligned}
$$

where $\psi_{i} \equiv \operatorname{Root}\left[8 \sharp 1^{4} c_{1}+\sharp 1^{3}\left(2 c_{2}-40 c_{1}\right)+\sharp 1^{2}\left(66 c_{1}+2 E\right)+\sharp 1\left(2 \mathrm{C}_{1}+2 \mathrm{C}_{2}-\right.\right.$ $\left.\left.40 c_{1}+2 c_{2}\right)+\mathrm{C}_{1}-\mathrm{C}_{2}+8 c_{1} \&, i\right](i=\overline{1,4})[1] ; z$ denotes the right part of equation (3.6), $c_{3}$ is an arbitrary constant, function $F$ is an elliptic integral of the first kind.

Although the function (3.7) has a sufficiently complicated form, its visualization is easily represented under a given set of parameters. For example, the plot of the solution

$$
x=\frac{F\left(\sin ^{-1}\left(\frac{2 y}{5}\right) \mid \frac{25}{36}\right) \sqrt{25-4 y^{2}} \sqrt{9-y^{2}}}{6 \sqrt{4 y^{4}-61 y^{2}+225}},
$$

which was obtained from the general solution of (3.7) for the following parameters:

$$
c_{1}=1, c_{2}=20, c_{3}=0, \mathrm{C}_{1}=221, \mathrm{C}_{2}=-221, E=-94,
$$

has the simple form (Fig. 1, continuous curve). These values of the parameters correspond to the initial condition $y(0)=0$ for the equation (1.11), where $z(y)$ is determined by the equation (3.6). We apply numerical integration to the Cauchy problem formulated in such a way and plot the corresponding graph (Fig. 1, dashed line). Figure 1 shows that the two curves (continuous and dashed) coincide.

Remark. Using the computer algebra systems for the analytical solutions (3.7) gives us the possibility to easily visualize the dependence of the six parameters $c_{1}, c_{2}, c_{3}, \mathrm{C}_{1}, \mathrm{C}_{2}, E$. We choose the certain values of these parameters and then can draw graph of the solution at the selected interval of variable $x$. For the root 


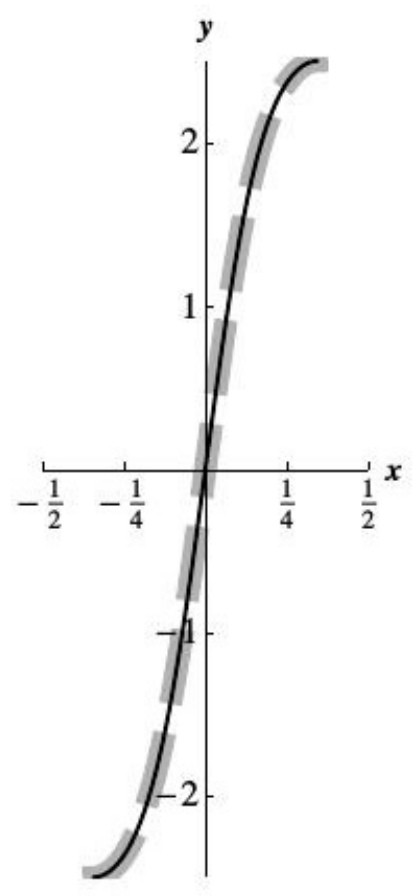

FIGURE 1. The graphs of the analytical and numerical solutions on the interval $[-1 / 2,1 / 2]$.

$\alpha_{22}=\frac{1}{8}(\sqrt{57}-11)$ coefficients $A_{k}, C_{k}$ have the form

$$
\begin{aligned}
& A_{1}=-\frac{1}{6}(6+\sqrt{57}), A_{2}=\frac{1}{6}(6+\sqrt{57}), \\
& A_{3}=\frac{1}{24}(\sqrt{57}-27), A_{4}=\frac{1}{24}(27-\sqrt{57}), \\
& A_{5}=\frac{1}{6}(3+\sqrt{57}), A_{6}=\frac{1}{6}(-3-\sqrt{57}), \\
& \mathrm{C}_{3}=\frac{9(7 \sqrt{57}-27) \mathrm{C}_{1}+(133-9 \sqrt{57}) \mathrm{C}_{2}+12(5 \sqrt{57}-7) E}{4(79+5 \sqrt{57})}, \\
& \mathrm{C}_{4}=\frac{(133-9 \sqrt{57}) \mathrm{C}_{1}+9(7 \sqrt{57}-27) \mathrm{C}_{2}+12(7-5 \sqrt{57}) E}{4(79+5 \sqrt{57})}, \\
& \mathrm{C}_{5}=\frac{-9(33+\sqrt{57}) \mathrm{C}_{1}+(127-17 \sqrt{57}) \mathrm{C}_{2}+(45 \sqrt{57}-321) E}{4(79+5 \sqrt{57})}, \\
& \mathrm{C}_{6}=\frac{(127-17 \sqrt{57}) \mathrm{C}_{1}-9(33+\sqrt{57}) \mathrm{C}_{2}+(321-45 \sqrt{57}) E}{4(79+5 \sqrt{57})},
\end{aligned}
$$


where $\mathrm{C}_{1}, \mathrm{C}_{2}, E$ are arbitrary parameters. The general solution of the corresponding linear equation (1.12) has the form

$$
\begin{aligned}
& z=c_{1} y\left(y^{2}+\frac{\sqrt{57}-11}{8}\right)+c_{2}\left(4(509+31 \sqrt{57}) y^{4}+\right. \\
& \left.(4941+903 \sqrt{57}) y^{2}-425 \sqrt{57}-3683\right)+ \\
& \frac{(3+4 \sqrt{57})\left(\mathrm{C}_{1}+\mathrm{C}_{2}\right) y+(7 \sqrt{57}-27)\left(\mathrm{C}_{1}-\mathrm{C}_{2}\right)}{79+5 \sqrt{57}}+E\left(y^{2}+\frac{2 \sqrt{57}-19}{7}\right),
\end{aligned}
$$

where $c_{1}, c_{2}$ are arbitrary constants. Substitute the relation (3.9) into formula (1.11). After integration we find the general solution of equation (1.9), (3.1), (3.8) using elliptic functions, similar to (3.7).

Under certain parameter sets the general solution can be expressed in the terms of elementary functions. For example, if

$$
\begin{aligned}
& E=-\frac{3}{8}(51+\sqrt{57}), \mathrm{C}_{1}=80+32 \sqrt{\frac{19}{3}}, \\
& \mathrm{C}_{2}=-80-32 \sqrt{\frac{19}{3}}, c_{1}=0, c_{2}=\frac{1}{2036+124 \sqrt{57}},
\end{aligned}
$$

then we obtain the one-parameter family of solutions of the form

$$
y=\frac{3-3 e^{6\left(c_{3}+x\right)}}{e^{6\left(c_{3}+x\right)}+1},
$$

where $c_{3}$ is arbitrary constant. 
For root $\alpha_{23}=-\frac{1}{8}(\sqrt{57}+11)$ coefficients $A_{k}, C_{k}$ have the form

$$
\begin{aligned}
& A_{1}=-1+\frac{\sqrt{57}}{6}, \quad A_{2}=1-\frac{\sqrt{57}}{6}, \\
& A_{3}=-\frac{\sqrt{57}+27}{24}, \quad A_{4}=\frac{27+\sqrt{57}}{24}, \\
& A_{5}=\frac{1}{6}(3-\sqrt{57}), \quad A_{6}=\frac{1}{6}(\sqrt{57}-3), \\
& \mathrm{C}_{3}=\frac{9(7 \sqrt{57}+27) \mathrm{C}_{1}-(133+9 \sqrt{57}) \mathrm{C}_{2}+12(5 \sqrt{57}+7) E}{4(5 \sqrt{57}-79)}, \\
& \mathrm{C}_{4}=\frac{(133+9 \sqrt{57}) \mathrm{C}_{1}-9(7 \sqrt{57}+27) \mathrm{C}_{2}+12(7+5 \sqrt{57}) E}{4(79-5 \sqrt{57})}, \\
& \mathrm{C}_{5}=\frac{9(\sqrt{57}-33) \mathrm{C}_{1}+(127+17 \sqrt{57}) \mathrm{C}_{2}-3(107+15 \sqrt{57}) E}{4(79-5 \sqrt{57})}, \\
& \mathrm{C}_{6}=\frac{(127+17 \sqrt{57}) \mathrm{C}_{1}-9(33-\sqrt{57}) \mathrm{C}_{2}+(321+45 \sqrt{57}) E}{4(79-5 \sqrt{57})},
\end{aligned}
$$

where $\mathrm{C}_{1}, \mathrm{C}_{2}, E$ are arbitrary parameters. The general solution of the corresponding linear equation (1.12) has the form

$$
\begin{gathered}
z=c_{1} y\left(y^{2}-\frac{\sqrt{57}+11}{8}\right)+c_{2}\left(4(509-31 \sqrt{57}) y^{4}+\right. \\
\left.(4941-903 \sqrt{57}) y^{2}+425 \sqrt{57}-3683\right)- \\
\frac{3+\sqrt{57}}{16}\left(\mathrm{C}_{1}+\mathrm{C}_{2}\right) y-\frac{6+\sqrt{57}}{7}\left(\mathrm{C}_{1}-\mathrm{C}_{2}\right)+E\left(y^{2}-\frac{2 \sqrt{57}+19}{7}\right),
\end{gathered}
$$

where $c_{1}, c_{2}$ are arbitrary constants. We substitute the relation (3.11) into formula (1.11). After integration we find the general solution of equation (1.9), (3.1), (3.10) using elliptic functions, similar to (3.7).

Let's now turn to the study of the multiple root $\alpha_{24}=-\frac{7}{4}$. Since the condition (1.6) for the root is performed, then to calculate coefficients $A_{k}, C_{k}$ we use formulas (1.7), (1.8). In this case, due to the multiplicity (is equal two) of the root, we will build two of the differential equations of the form (1.9) for which the corresponding 
coefficients $A_{k}$ have the form

$$
\begin{gathered}
A_{1}=-1+\sqrt{\frac{11}{6}}, A_{2}=1+\sqrt{\frac{11}{6}}, \\
A_{3}=-\frac{36+\sqrt{66}}{30}, A_{4}=\frac{36-\sqrt{66}}{30}, \\
A_{5}=\frac{12-2 \sqrt{66}}{15}, A_{6}=-\frac{2}{15}(6+\sqrt{66}), \alpha_{3}=-\frac{1}{4} \sqrt{\frac{33}{2}}
\end{gathered}
$$

or

$$
\begin{gathered}
A_{1}=-1-\sqrt{\frac{11}{6}}, A_{2}=1-\sqrt{\frac{11}{6}}, \\
A_{3}=\frac{\sqrt{66}-36}{30}, A_{4}=\frac{36+\sqrt{66}}{30}, \\
A_{5}=\frac{12+2 \sqrt{66}}{15}, A_{6}=\frac{2}{15}(\sqrt{66}-6), \alpha_{3}=\frac{1}{4} \sqrt{\frac{33}{2}} .
\end{gathered}
$$

Sets of coefficients (3.12) and (3.13) define the two different types of equations (1.9).

Substituting coefficients (3.1), (3.12) into system (1.10) and solving it, we calculate

$$
\begin{aligned}
& \mathrm{C}_{3}=\frac{-9(6+\sqrt{66}) \mathrm{C}_{1}+(\sqrt{66}-14) \mathrm{C}_{2}-6(\sqrt{66}-4) E}{40}, \\
& \mathrm{C}_{4}=\frac{-(14+\sqrt{66}) \mathrm{C}_{1}+9(\sqrt{66}-6) \mathrm{C}_{2}-6(4+\sqrt{66}) E}{40}, \\
& \mathrm{C}_{5}=\frac{9(\sqrt{66}-6) \mathrm{C}_{1}-(14+\sqrt{66}) \mathrm{C}_{2}-6(4+\sqrt{66}) E}{160}, \\
& \mathrm{C}_{6}=\frac{(\sqrt{66}-14) \mathrm{C}_{1}-9(6+\sqrt{66}) \mathrm{C}_{2}-6(\sqrt{66}-4) E}{160},
\end{aligned}
$$

where $\mathrm{C}_{1}, \mathrm{C}_{2}, E$ are arbitrary parameters. The general solution of the corresponding linear equation (1.12) has the form

$$
\begin{gathered}
z=c_{1}\left(y^{2}+1\right)\left(\sqrt{11} y^{2}-\sqrt{6} y-\sqrt{11}\right)+ \\
c_{2}\left(11-\sqrt{57}-8 y^{2}\right)(y((\sqrt{66}+\sqrt{418}) y-44)+ \\
\sqrt{418}-\sqrt{66})+\mathrm{C}_{1}\left(-\frac{1}{8} \sqrt{\frac{33}{2}}(y+1)-\frac{3}{16}(2 y+1)\right)+ \\
\frac{1}{16} \mathrm{C}_{2}((\sqrt{66}-6) y-\sqrt{66}+3)+E\left(y^{2}-\frac{1}{8}(\sqrt{66} y+11)\right),
\end{gathered}
$$

where $c_{1}, c_{2}$ are arbitrary constants. Substituting relation (3.15) into formula (1.11) and integrating the obtained equation, we find the general solution of equation (1.9), (3.1), (3.12), (3.14) using the elliptic functions in the implicit form, similar to (3.7). 
Set of coefficients (3.13) corresponds to the following values of the coefficients $\mathrm{C}_{k}$

$$
\begin{aligned}
& \mathrm{C}_{3}=\frac{9(\sqrt{66}-6) \mathrm{C}_{1}-(\sqrt{66}+14) \mathrm{C}_{2}+6(\sqrt{66}+4) E}{40}, \\
& \mathrm{C}_{4}=\frac{(\sqrt{66}-14) \mathrm{C}_{1}-9(\sqrt{66}+6) \mathrm{C}_{2}+6(\sqrt{66}-4) E}{40}, \\
& \mathrm{C}_{5}=\frac{-9(\sqrt{66}+6) \mathrm{C}_{1}+(\sqrt{66}-14) \mathrm{C}_{2}+6(\sqrt{66}-4) E}{160}, \\
& \mathrm{C}_{6}=\frac{-(\sqrt{66}+14) \mathrm{C}_{1}+9(\sqrt{66}-6) \mathrm{C}_{2}+6(\sqrt{66}+4) E}{160}
\end{aligned}
$$

and the corresponding differential equation is integrated in a similar way; its solution is built in the implicit form, too.

\section{CONCLUSiOnS}

The algorithm to determine the coefficients of the nonlinear third-order differential equation with six constant poles with respect to the unknown function - that satisfy the system of algebraic equations - is described. Five differential equations are integrated for the case when the roots of the corresponding algebraic equation of the fifth degree are expressed in radicals. Their general solutions are obtained using the elliptic functions and depend on three parameters and three arbitrary constants of integration.

It should be noted that the computational algorithm for the construction of differential equations and their integration procedure are connected with carrying out of cumbersome calculations, which have been performed using a computer algebra system Mathematica.

The results can be used in the study of the equation (1.3) in the case when the coefficients $a_{k}$ are not constants.

The author is grateful to Professor A.N. Prokopenya for useful discussion and valuable comments. 


\section{APPLICATION}

The system of algebraic and differential equations determines the coefficients of the equation (1.3):

$$
\begin{aligned}
& \sum_{k=1}^{6} A_{k}=0, \sum_{k=1}^{6} a_{k} A_{k}=-6, \\
& \sum_{k=1}^{6} a_{k}^{2} A_{k}=-2 \sum_{k=1}^{6} a_{k} \\
& 2 A_{k}^{2}+\sum_{j} \frac{A_{k}-A_{j}}{a_{k}-a_{j}}=0(k, j=\overline{1,6} ; k \neq j), \\
& \left(\frac{5}{2} A_{k}-\sum_{j} \frac{1}{a_{k}-a_{j}}\right) B_{k}+\sum_{j}\left(\frac{1}{2} A_{k}+\frac{1}{a_{k}-a_{j}}\right) B_{j}= \\
& -A_{k}^{\prime}+A_{k} \sum_{j} \frac{a_{k}^{\prime}-a_{j}^{\prime}}{a_{k}-a_{j}}-3 \sum_{j} A_{j} \frac{a_{k}^{\prime}-a_{j}^{\prime}}{a_{k}-a_{j}}+\frac{3}{2} A_{k} \sum_{i=1}^{6} a_{i}^{\prime} A_{i}, \\
& 2 D+\sum_{k=1}^{6}\left(B_{k}-3 a_{k}^{\prime} A_{k}\right)=0, \\
& -\left(2 A_{k}+\sum_{j} \frac{1}{a_{k}-a_{j}}\right) \mathrm{C}_{k}+\sum_{j} \frac{\mathrm{C}_{j}}{a_{k}-a_{j}}=B_{k}^{2}-B_{k}^{\prime}- \\
& B_{k} \sum_{j} \frac{a_{k}^{\prime}-a_{j}^{\prime}}{a_{k}-a_{j}}-\sum_{j} \frac{3 A_{j}\left(a_{k}^{\prime}-a_{j}^{\prime}\right)^{2}+2 B_{j}\left(a_{k}^{\prime}-a_{j}^{\prime}\right)}{a_{k}-a_{j}}+ \\
& B_{k} D-E-\sum_{j} \frac{a_{k}^{\prime \prime}-a_{j}^{\prime \prime}}{a_{k}-a_{j}}, \\
& -a_{k}^{\prime \prime \prime}-B_{k} \mathrm{C}_{k}+\mathrm{C}_{k}^{\prime}+\sum_{j} \frac{\left(a_{k}^{\prime}-a_{j}^{\prime}\right)\left(a_{k}^{\prime \prime}-a_{j}^{\prime \prime}-\mathrm{C}_{k}\right)}{a_{k}-a_{j}}+ \\
& \sum_{j} \frac{\left.A_{j}\left(a_{k}^{\prime}-a_{j}^{\prime}\right)^{3}+B_{j}\left(a_{k}^{\prime}-a_{j}^{\prime}\right)^{2}+\mathrm{C}_{k}\right)\left(a_{k}^{\prime}-a_{j}^{\prime}\right)}{a_{k}-a_{j}}+ \\
& E a_{k}^{\prime}+D\left(a_{k}^{\prime \prime}-\mathrm{C}_{k}\right)+F_{k} \prod_{j}\left(a_{k}-a_{j}\right)=0,
\end{aligned}
$$




$$
\sum_{k=1}^{6} F_{k}=\sum_{k=1}^{6} a_{k} F_{k}=\sum_{k=1}^{6} a_{k}^{2} F_{k}=0,
$$

where $k, j=\overline{1,6} ; k \neq j$.

\section{REFERENCES}

[1] Wolfram Language \& System Documentation Center. [Online]. Available: http://reference. wolfram.com/language/ref/Root.html

[2] M. L. Abell and J. P. Braselton, Differential equations with Mathematica. Burlington: Elsevier, 2004.

[3] S. A. Abramov, Elements of computer algebra for the linear ordinary differential, difference and q-difference operators. Moscow: MCCME, 2012, (in Russian).

[4] J. Chazy, "Sur les equations differentielles du troisieme ordre et dordre superieur, dont lintegrale generale a ses points critiques fixes," Acta Math., vol. 34, pp. 317-385, 1911, doi: 10.1007/BF02393131.

[5] A. Chichurin, "Investigation of a class of the Chazy equations," Bulletin of Taras Shevchenko National University of Kyiv (Series Mathematics \& Mechanics), vol. 24, pp. 14-20, 2010, (in Ukranian).

[6] A. V. Chichurin, The Chazy equation and linear equations of the Fuchs class. Moscow: RUDN, 2003, (in Russian).

[7] A. V. Chichurin, "The problem of existence of mappings between classes of the Chazy equations and second-order equations," Vesnik of the Belarusian University. Series 1, Mathematics, vol. 21, no. 2, pp. 74-78, 2003, (in Russian).

[8] A. V. Chichurin, "On exact solutions of the nonlinear third-order differential equation with six singular points," Dynamics of inhomogeneous systems, Proceedings of ISA RAS., vol. 56, pp. 20 29, 2010, (in Russian).

[9] A. V. Chichurin, "Solution the Chazy system and integration of a Chazy differential equation with six constant poles by means of the Mathematica system," Vesnik of Brest University. Series 4. Physics. Mathematics, no. 2, pp. 134-141, 2010, (in Russian).

[10] A. V. Chichurin and H. H. Shvychkina, "On the integrability of the third-order systems, that are equivalent to the Chazy equation with six fixed poles," I. Yakovlev Chuvash State Pedagogical University Bulletin, Series: Mechanics of a limiting condition., vol. 4, no. 22, pp. 176-185, 2014, (in Russian).

[11] V. Dobrovolsky, Essays on the development of the analytic theory of differential equations. Kiev: Vishcha shkola, 1974, (in Russian).

[12] N. A. Lukashevich, "The equations of the third order without movable critical points (m.c.p.)," Differ. Equ., vol. 18, no. 5, pp. 778-785, 1982.

[13] N. A. Lukashevich, "To the Chazy equation theory," Differ. Equ., vol. 29, no. 2, pp. 353-357, 1993.

[14] I. P. Martynov and A. V. Chichurin, "On a solution of the Chazy system of equations," Nonlinear Oscillations., vol. 12, no. 1, pp. 94-100, 2009, doi: 10.1007/s11072-009-0062-8.

[15] V. N. Orlov, The method of approximate solution for the first, the second Painleve and the Abel differential equations. Moscow: MSPU, 2013, (in Russian).

[16] P. Painleve, Lecons sur les theorie analytique des equations differentielles. Professies a Stokholm, Paris, 1897.

[17] A. N. Prokopenya and A. V. Chichurin, Application of the Mathematica system to the solution of ordinary differential equations. Minsk: BSU, 1999, (in Russian). 
[18] H. Shvychkina, "Building the third-order differential system with Mathematica," Computer Algebra Systems in Teaching and Research. Differential Equations, Dynamical Systems and Celestial Mechanics., pp. 136-140, 2011.

\section{Author's address}

\section{Alexander Chichurin}

Computer modeling and simulation department, The John Paul II Catholic University of Lublin, ul. Konstantynow 1H, 20-708 Lublin, Poland

E-mail address: achichurin@gmail.com 